\title{
Oclusão de Fístula Arteriovenosa Pulmonar com Plug Vascular em Paciente com Embolia Cerebral Paradoxal Prévia
}

\author{
Dárcio Gitti de Faria1', Pedro Gomes de Almeida Garzon¹, José Luiz Balthazar Jacob
}

\section{RESUMO}

A embolização percutânea transcateter tornou-se o tratamento de escolha para fístulas arteriovenosas pulmonares (FAVPs), na maioria dos casos em substituição à intervenção cirúrgica. Os dispositivos clássicos, tais como coils intravasculares e balões destacáveis, provaram ser um sucesso para oclusões percutâneas de FAVPs pequenas ou médias; porém, não são ideais nas fístulas maiores e de alto fluxo pelo risco de embolização para a circulação sistêmica. Relatamos o caso de uma mulher de 49 anos de idade com FAVP de alto fluxo e manifestações neurológicas isquêmicas prévias, submetida com sucesso a fechamento percutâneo com Amplatzer ${ }^{\text {TM }}$ Vascular Plug II, um dispositivo projetado para oclusão de anormalidades vasculares.

DESCRITORES: Fístula arteriovenosa. Artéria pulmonar. Embolização terapêutica. Oclusão coronária.

\begin{abstract}
Occlusion of Pulmonary Arteriovenous Fistula

Using Vascular Plug in a Patient with Previous Paradoxal Cerebral Embolism

Percutaneous transcatheter embolization has become the treatment of choice for pulmonary arteriovenous fistulae (PAVFs), replacing surgical intervention in most cases. Classical devices, such as intravascular coils and detachable balloons, have proven to be successful for percutaneous occlusions of small or medium-sized PAVFs; however, they are not ideal for larger and high flow fistulae due to the risk of embolization to systemic circulation. The case of a 49-year-old woman with high flow pulmonary fistula and previous neurological ischemic manifestations undergoing successful transcatheter closure with an Amplatzer ${ }^{\mathrm{TM}}$ Vascular Plug II, a device designed for occlusion of vascular abnormalities, is reported.
\end{abstract}

KEY-WORDS: Arteriovenous fistula. Pulmonary artery. Embolization, therapeutic. Coronary occlusion.

Vascular Plug II (AGA Medical Corporation, Golden Valley, Estados Unidos).

\section{RELATO DO CASO}

Paciente do sexo feminino, com 49 anos de idade, apresentou quadro de paresia e parestesia em membro superior direito há três anos, que regrediu em 24 horas, sendo atendida em outro serviço em que foi confirmado diagnóstico de AIT por meio de ressonância nuclear magnética. Ainda nesse atendimento foi realizada radiografia de tórax, evidenciando duas imagens nodulares com densidade de partes moles e limites precisos (> $8 \mathrm{~mm}$ ) em base pulmonar esquerda. O mesmo quadro clínico se repetiu três meses depois. Em decorrência do aspecto radiológico confirmado nesse segundo episódio de AIT, a paciente foi avaliada por pneumologista, que levantou a hipótese diagnóstica de malformação arteriovenosa pulmonar, sendo encaminhada para o serviço de cirurgia vascular. Após período longo de avaliação e medicação com varfarina, foi encaminhada a nossa instituição.
'Serviço de Hemodinâmica do Instituto de Moléstias Cardiovasculares - São José do Rio Preto, SP, Brasil.

Correspondência: Dárcio Gitti de Faria. Rua Castelo D'Água, 3030 Redentora - São José do Rio Preto, SP, Brasil - CEP 15015-210 E-mail: darciogfaria@ig.com.br

Recebido em: 8/12/2010 • Aceito em: 15/2/2011 
Em atendimento ambulatorial, a paciente não referiu sintomas e o exame físico não demonstrou alterações. Estava em uso de varfarina, mantendo nível adequado de anticoagulação. A pesquisa de trombofilia e distúrbios da coagulação foram negativos e a ecocardiografia Doppler de carótidas e vertebrais não revelou anormalidades. A radiografia de tórax confirmou as imagens nodulares descritas anteriormente (Figura 1). Ecocardiograma transesofágico demonstrou: função sistólica preservada dos ventrículos esquerdo e direito, com átrio esquerdo de tamanho normal, sem evidência de trombo ou contraste espontâneo em seu interior, inclusive em apêndice atrial; veias pulmonares drenando para o átrio esquerdo; e ausência de forame oval patente ou shunts intracardíacos. Após a infusão de microbolhas, detectou-se a presença de microbolhas no átrio esquerdo, em grande quantidade, após o quarto batimento cardíaco, sugerindo a presença de shunt arteriovenoso pulmonar, que poderia corresponder às imagens visíveis no estudo radiológico do tórax.

Foi indicado e realizado cateterismo cardíaco, com angiografias de artérias pulmonares direita e esquerda, que demonstraram a presença de fístula arteriovenosa pulmonar com formação de grande lago venoso trilobulado, no lobo inferior do pulmão esquerdo, decorrente de ramo arterial secundário da artéria pulmonar, com $7 \mathrm{~mm}$ de diâmetro que se bifurcava e se comunicava diretamente com as veias pulmonares, determinando shunt de alto fluxo (Figura 2A). Diante disso, optou-se pelo fechamento percutâneo com Amplatzer ${ }^{\mathrm{TM}}$ Vascular Plug II há três anos.

O procedimento foi realizado sob sedação anestésica, por punção de veia femoral direita, sendo posicionado introdutor $7 \mathrm{~F}$. Foram administradas heparina $(100 \mathrm{UI} / \mathrm{kg})$ e cefalotina $(25 \mathrm{mg} / \mathrm{kg})$. Pelo introdutor $7 \mathrm{~F}$ foi avançado um cateter-guia de angioplastia de coronária direita JR $7 \mathrm{~F}$, posicionado no tronco da artéria

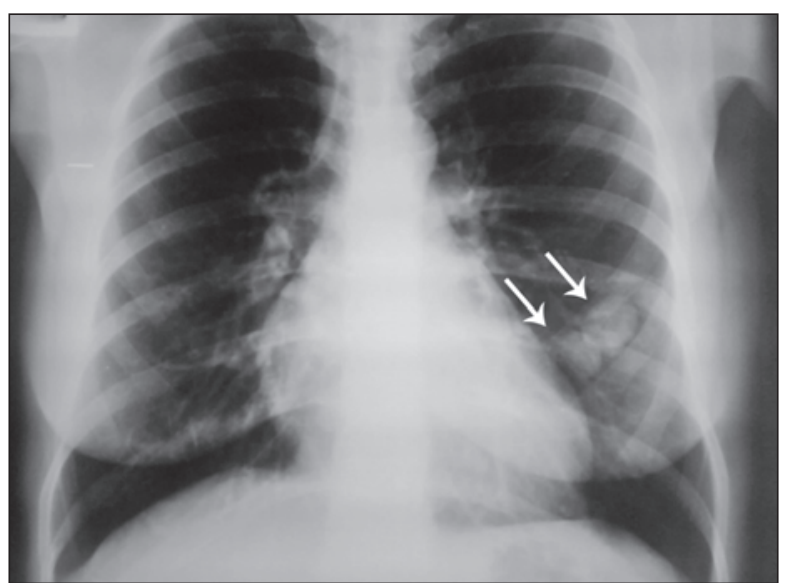

Figura 1 - Radiografia de tórax com duas imagens nodulares (setas) com densidade de partes moles em base pulmonar esquerda, correspondentes ao lago venoso trilobulado. pulmonar. Com o auxílio de um fio-guia, o cateter foi avançado até o ramo da artéria pulmonar esquerda, que determinava a conexão arteriovenosa pulmonar anômala, com formação do lago venoso trilobulado. Pelo cateter-guia de angioplastia JR foi avançado um Amplatzer $^{\text {TM }}$ Vascular Plug II de $10 \mathrm{~mm}$ e seu disco distal foi liberado na extremidade distal do ramo arterial, próximo à área de bifurcação que desembocava no lago venoso, puxando-se o cateter e expondo o dispositivo. A angiografia em artéria pulmonar esquerda demonstrou ausência completa de passagem de contraste da artéria para o lago venoso pulmonar, com oclusão total da FAVP (Figura 2B). A paciente foi mantida com clopidogrel (75 mg/dia), ácido acetilsalicílico (325 mg/dia) e cefalexina (500 mg a cada 6 horas por 5 dias). Evoluiu sem intercorrências, recebendo alta hospitalar no dia seguinte. Os ecocardiogramas transtorácicos com infusão de microbolhas realizados após um mês e seis meses do procedimento não evidenciaram presença de bolhas no átrio esquerdo.

\section{DISCUSSÃO}

FAVPs são conexões anormais diretas entre as circulações arterial e venosa, interpostas por um saco aneurismático ou um emaranhado de canais vasculares tortuosos e dilatados, com exclusão do leito capilar. ${ }^{3}$ Essa infrequente anomalia vascular pode ser isolada ou múltipla. ${ }^{2}$ A forma congênita tem duas variedades: a) angioma cavernoso, geralmente alimentado por um ou mais ramos tortuosos e dilatados da artéria pulmonar; e b) telangiectasia capilar, formada por uma rede de capilares, geralmente associada a telangiectasia hemorrágica hereditária ou síndrome de Rendu-Osler-Weber (displasia vascular autossômica dominante com penetrância variável, caracterizada por múltiplas telangiectasias, causando hemorragias recorrentes). ${ }^{4}$

Mais de $80 \%$ das FAVPs são congênitas, enquanto as formas adquiridas são secundárias a trauma cirurgia torácica, cirrose hepática avançada, carcinoma metastático, estenose mitral e amiloidose sistêmica. ${ }^{5,6}$ A FAVP descrita neste relato apresentava etiologia congênita do tipo angioma cavernoso.

Aproximadamente $57 \%$ dos pacientes são assintomáticos. ${ }^{7}$ Os sinais e sintomas mais comuns são fadiga, dispneia de esforço, palpitações, cianose e hemoptise, sendo a dispneia o mais frequente. ${ }^{8}$ Complicações graves como abscesso cerebral, acidente vascular encefálico ou acidentes isquêmicos cerebrais transitórios, como os apresentados por essa paciente, são relatados. ${ }^{1,2}$ Ocasionalmente, durante o exame físico, um sopro contínuo extracardíaco pode ser ouvido na ausculta do tórax, ${ }^{2}$ o que não foi encontrado no exame físico da paciente.

Para o diagnóstico, a radiografia de tórax revela mais de $98 \%$ das lesões. ${ }^{5}$ Apesar de o ecocardiograma transtorácico com infusão de microbolhas ser adequa- 

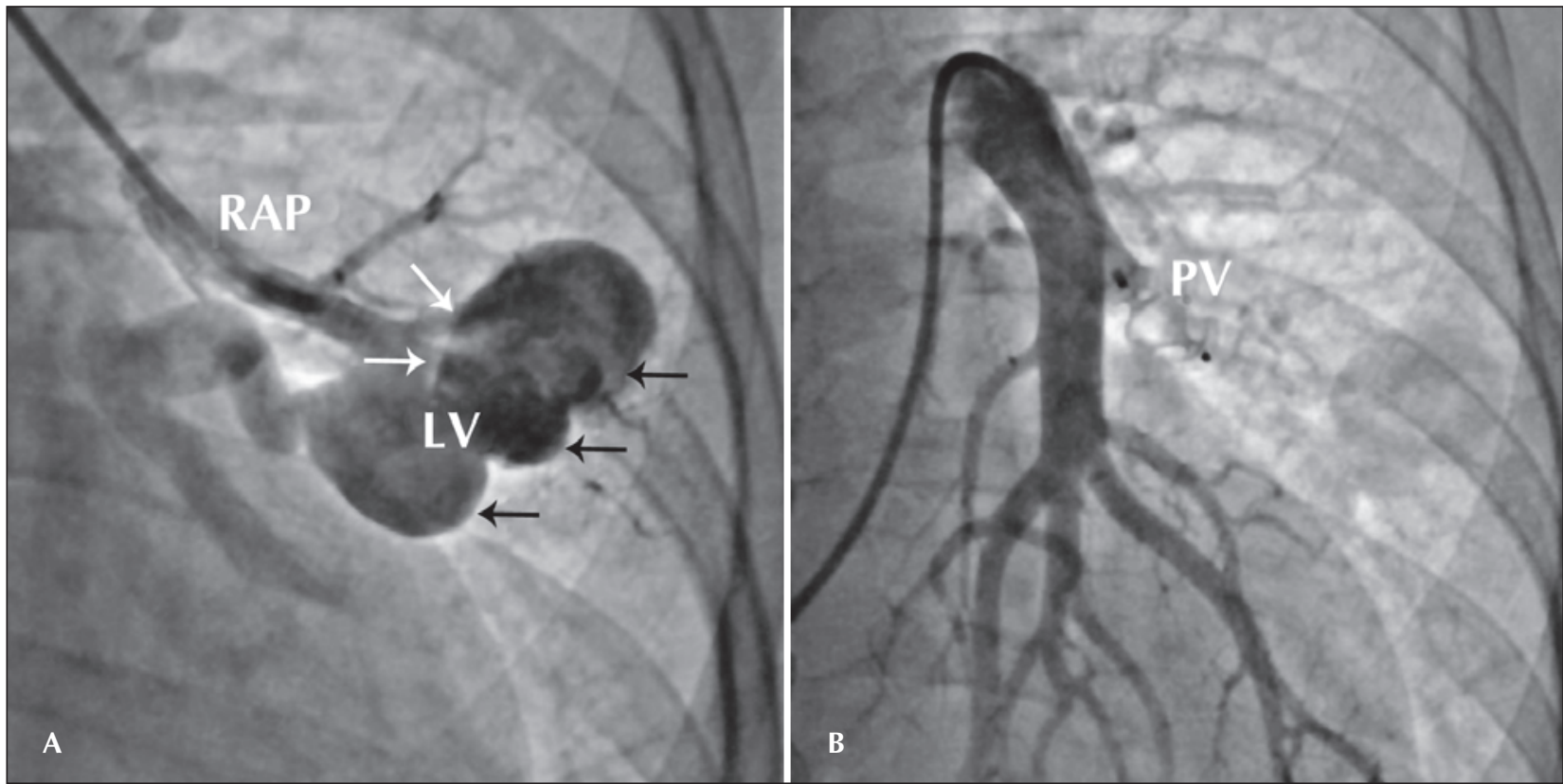

Figura 2 - Em A, bifurcação (setas brancas) do ramo arterial pulmonar (RAP), dando origem à fístula arteriovenosa pulmonar com formação de grande lago venoso (LV) trilobulado (setas pretas). Em B, oclusão total de fístula arteriovenosa pulmonar com plug vascular (PV).

do para documentar um shunt intracardíaco ou intrapulmonar, a visibilização seletiva das veias pulmonares pode ser obtida com o ecocardiograma transesofágico e o teste de microbolhas pode determinar a localização do shunt intrapulmonar. ${ }^{7}$ O surgimento tardio de microbolhas no átrio esquerdo, depois do quarto ou do quinto ciclos cardíacos, é consistente com shunt extracardíaco, ${ }^{4,5,7}$ como evidenciado no caso descrito neste relato, em que grande quantidade de microbolhas surgiu a partir do quarto e quinto batimentos cardíacos.

A tomografia computadorizada e a ressonância magnética também são de grande valor diagnóstico, ${ }^{9}$ porém não foram realizadas nessa paciente. A angiografia é crucial para confirmar o diagnóstico e definir a estratégia terapêutica. ${ }^{2,9}$

As opções de tratamento incluem a embolização percutânea com coils, balão destacável ou outros dispositivos e a excisão cirúrgica. ${ }^{3}$ Embora a embolização com coils seja considerada o tratamento de escolha, complicações podem ocorrer. ${ }^{8}$ A embolização paradoxal do dispositivo varia numa taxa de $2 \%$ a $4 \%$, especialmente quando a FAVP é grande e tem alto fluxo. ${ }^{10}$ Recorrência é secundária à recanalização dos vasos ocluídos ou ao desenvolvimento de vasos acessórios. ${ }^{8}$ Recanalização é a causa mais comum de recorrência, incidindo numa taxa de 5\% a 57\%. ${ }^{11}$

A ressecção cirúrgica é reservada aos pacientes com falha na terapia percutânea e aos que desenvolveram graves complicações hemorrágicas ou com lesões inapropriadas para oclusão percutânea. ${ }^{12}$
A escolha adequada e precisa do material a ser utilizado para o fechamento percutâneo é importante para diminuir o insucesso do procedimento e as complicações. Na paciente deste relato, portadora de grande FAVP de alto fluxo, optou-se pelo uso do dispositivo Amplatzer ${ }^{\mathrm{TM}}$ Vascular Plug II, especialmente desenvolvido para oclusão de estruturas vasculares anormais, obtendo-se sucesso no procedimento, mantido nos primeiros seis meses de acompanhamento.

\section{CONFLITO DE INTERESSES}

Os autores declaram não haver conflito de interesses relacionado a este manuscrito.

\section{REFERÊNCIAS}

1. Rossi M, Rebonato A, Greco L, Stefanini J, Citone M, Speranza $A$, et al. A new device for vascular embolization: report on case of two pulmonary arteriovenous fistulas embolization using the Amplatzer Vascular Plug. Cardiovasc Intervent Radiol. 2006;29(5):902-6.

2. Bialkowski J, Zabal C, Szkutnik M, Montes JAG, Kusa J, Zembala M. Percutaneous interventional closure of a large pulmonary arteriovenous fistulas with Amplatzer Duct Occluder. Am J Cardiol. 2005;96(3):127-9.

3. Ferro C, Rossi UG, Bovio G, Seitun S, Rossi GA. Percutaneous transcatheter embolization of a large pulmonary arteriovenous fistula with an Amplatzer Vascular Plug. Cardiovasc Intervent Radiol. 2007;30(8):328-31.

4. Gianisella RB, Rossi Filho RI, Zielinsky P. Diagnosis and therapeutics of pulmonary arteriovenous fistula in childhood. Case report and review of the literature. Arq Bras Cardiol. $2001 ; 77(3): 278-81$

5. Tomelleri G, Bovi P, Carletti M, Mazzucco S, Bazzoli E, Casilli $F$, et al. Paradoxal brain embolism in a young man 
with isolated pulmonary arteriovenous fistula. Neurol Sci. 2008;29(4):169-71.

6. Khurshid I, Downie GH. Pulmonary arteriovenous malformation. Postgrad Med J. 2002;78(10):191-7.

7. Oliveira GHM, Seward JB, Stanson AW, Swanson JW. Paradoxal cerebrovascular embolism associated with pulmonary arteriovenous fistula: contrast transoesophageal echocardiographic diagnosis. Eur J Echocardiogr. 2001;2(3): 207-11.

8. Abdel Aal AK, Hamed MF, Biosca RF, Saddekni S, Raghuram K. Occlusion time for Amplatzer Vascular Plug in the management of pulmonary arteriovenous malformations. AJR Am J Roentgenol. 2009;192(3):793-9.
9. De Castro CR, Ikari NM, Jatene FB. Pulmonary arteriovenous fistula. Heart. 2003;89(1):41.

10. Lee DW, White RI, Egglin TK, Pollak JS, Fayad PB, Wirth JA, et al. Embolotherapy of large pulmonary arteriovenous malformations: long-term results. Ann Thorac Surg. 1997; 64(4):930-40.

11. Magger JJ, Overtoom TT, Blauw H, Lammeers JW, Westermann C). Embolotherapy of pulmonary arteriovenous malformations: long-term results in 112 patients. J Vasc Interv Radiol. 2004; 15(5):451-6.

12. Zhang SY, Zhang ZT, Ou SL, Hu YS, Song FQ, Li X, et al Fistulectomy as a surgical option for pulmonary arteriovenous malformation. Chin Med J (Engl). 2009;122(19):2321-4. 